Manuelle Medizin 2021 · 59:79-80

https://doi.org/10.1007/s00337-021-00777-8

Angenommen: 24. Februar 2021

(c) Springer Medizin Verlag GmbH, ein Teil von Springer Nature 2021

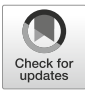

L. Beyer

Ärztehaus Mitte, Jena, Deutschland

\title{
Denken in Schlüsselfunktionen: spezifische funktionelle Herangehensweisen
}

der Beitrag von Zwingenberger, in dem er eine „historische Betrachtung zur Annahme der dominierenden manualtherapeutischen Schlüsselregionen “ anstellt und zu diesem Begriff und seiner Verwendung recherchiert hat. Er postuliert: „Der anhaltende Erfolg einer manualtherapeutischen Intervention hängt im allgemeinen Konsensus von der alleinigen oder zumindest Mitbehandlung der sog. Schlüsselregionen ab.“

Bei meinen eigenen Nachforschungen fand ich, dass die sog. Übergänge kraniozervikal, zervikothorakal und lumbosakral häufig als Schlüsselregionen bezeichnet werden, wobei meist unklar bleibt, ob damit nur die Wirbelsäule oder z.B. beim zervikothorakalen Übergang die Nacken-Schulter-Region als Ganzes inklusive Wirbelsäule gemeint ist. Häufig behandelte Strukturen sowohl in der manuellen Medizin als auch Osteopathie sind der Beckenboden, das Iliosakralgelenkoder ein Beckenschiefstand. So wundert es nicht, dass uns in der vorliegenden Ausgabe ein Beitrag von Ritzmann vorliegt: „Schlüsselregion Becken - Krankheitsbilder einer gynäkologisch-geburtshilflichen Praxis“. Die Autorin postuliert: „Das Becken ist eine Schlüsselregion für die manuelle Behandlung verschiedenster Beschwerden, auch aus dem gynäkologischen und geburtshilflichen Bereich, Beckenringdysfunktionen sind häufig." Den Charakter der Schlüsselregion Becken und verallgemeinert für die Definition dessen, was eine Schlüsselregion ausmachen könnte, beschreibt sie in ihrem Beitrag so: „Das Becken bleibt lebenslang ein wichtiges Zentrum der gesamten muskulären Koordination und der Skelettsymmetrie. Als Schlüsselregion be- herbergt das Becken ein dichtes Netz von motorischen, sensiblen, sympathischen und parasympathischen Nervenfasern, die bei Fehlstellungen des Beckenrings neben viszeralen Funktionsstörungen von Dickdarm, Harnblase und Genitalien auch einen entscheidenden Einfluss auf Dysfunktionen von Wirbelsäule, Nacken, Schultern, Hüfte und den Extremitäten haben können. Die manuelle Behandlung einer Beckenringdysfunktion bei gynäkologischen Problemen hat so oft eine günstige Fernwirkung." Im Beitrag werden anatomische, funktionelle und epidemiologische Zusammenhänge analysiert, Beispiele von Dysfunktionen und Beschwerden sind dargestellt, diagnostisches Vorgehen und Therapieansätze werden aufgezeigt.

In die durch den Begriff „Schlüsselregion“ aufgezeigte Problematik „funktionelle Verknüpfungen in einer Region und davon ausgehende Fernwirkung (oft nicht ganz klar als Verkettungen bezeichnet)" fügt sich auch die Publikation nach einem Vortrag auf dem ZIMMTKongress von Wuttke: „HNO-Aspekte bei kindlicher Mundatmung und Kieferfehlstellungen“" ein. Im Vordergrund stehen die kindliche Atmung und ihre Beeinflussung bzw. sekundäre Störung durch morphologische Parameter und primäre Störungen im Bereich Lippen, Mundhöhle, Muskulatur, Kiefergelenk und Wirbelsäule, die er als Funktionslogen bezeichnet, man könnte sagen, in einer gemeinsamen „kraniomandibulären“ Schlüsselregion. So schreibt Wuttke: „Der Einfluss eines eugnathen Kau-Kiefer-Systems auf die Entwicklung und der funktionelle Zusammenhang mit anderen Komponenten des Bewegungs- 
und Haltesystems sowie die gemeinsame Bedeutung für eine orthograde und physiologische Entwicklung unterstreichen die Wichtigkeit in der frühzeitigen Diagnostik/Frühintervention."

Wie auch bei den vorher genannten Beiträgen steht bei Dürrschnabel u. Dürrschnabel „Gleichgewichtssinn und Symmetrie - untrennbare Einheit einer stabilen Bipedalität" die Funktionalität komplexer Bewegungsabläufe im Mittelpunkt der Beschreibung des Ganges. Interessant ist der Vorschlag eines über das Elektromyogramm (EMG) zu ermittelnden Kokontraktionsindex für Aussagen über die ipsilaterale und kontralaterale Muskelaktivität. Unter biomechanischen Darlegungen zum Gang wird dem Fuß eine Schlüsselrolle für die Stabilität im Raum zugeordnet.

In einer Literaturrecherche sichtete Thiele 1046 Artikel und fand 169 zum Thema „chiropraktische Behandlung bei unteren Rückenschmerzen“, davon 54 systematische Übersichtsarbeiten und 115 randomisierte klinische Studien, von denen 13 RCT für einen direkten Therapievergleich für die Übersicht geeignet waren. Als Hauptaussage ergab sich, dass im direkten Vergleich mit der McKenzie-Therapie bessere Ergebnisse für die McKenzie erreicht wurden. Die Übersicht spiegelt den aktuellen Stand der Behandlung von unterem Rückenschmerz mit dieser der manuellen Medizin zuzuordnenden Methode wider.

Entsprechend unserem Konzept, die Zeitschrift auch im europäischen Rahmen sichtbar zu machen, wird dieser Beitrag zusätzlich auch in Englisch als Onlineversion erscheinen.

\section{Das Coronavirus - Neben- wirkungen auf unsere MM- Gesellschaft}

Bedingt durch die Coronapandemie haben sich natürlich auch Zwänge für das wissenschaftliche Leben in unseren Gesellschaften ergeben. So wurde der jährlich stattfindende ZIMMT-Kongress erst verschoben, dann aber hervorragend organisiert als Videokonferenz durchgeführt. Nachteil: Es fehlt der direkte Kontakt; Vorteil: Man kann die einzelnen
Beiträge auch noch einige Wochen nach dem Kongresstermin in Ruhe anhören. Eine Übersicht der Kongressbeiträge finden Sie in „mm - aktuell“.

Die Weiterbildung in manueller Medizin kam zeitweilig zum Erliegen oder muss unter verschärften Hygienebedingungen durchgeführt werden. Erste Versuche mit Videoübertragungen wurden gestartet. Auch hier wird die Pandemie das Nachdenken und die Realisierung der Einführung digitaler Medien in die Kursgestaltung beschleunigen. Beyer $u$. Winkelmann stellen dazu im Beitrag „Digitale Transformation der manualmedizinischen und manualtherapeutischen Weiterbildung - Integration digitaler Medien in den motorischen Lernprozess" zur Diskussion.

Bereits im letzten Heft hatten wir die Darstellung von Techniken wieder aufgenommen. Dies soll einerseits die Diskussion zwischen verschiedenen Lehrmeinungen und in der täglichen Praxis Erfahrenen anstoßen, andererseits aber auch an Beispielen zeigen, welche Genauigkeit erforderlich ist, um zu beschreiben, was wir tun, nicht zuletzt auch bei der Schilderung manueller Untersuchung und Behandlung in wissenschaftlichen Veröffentlichungen, da dies immer wieder als ungenügend bemängelt wird.

$\mathrm{Zu}$ guter Letzt sei darüber informiert, dass auch die Mitgliederversammlung der DGMM in einer Videokonferenz durchgeführt wurde. Als neuer Präsident wurde Prof. Hermann Locher gewählt. Wir wünschen ihm viel Erfolg und Schaffenskraft für unsere gemeinsame Sache der manuellen Medizin.

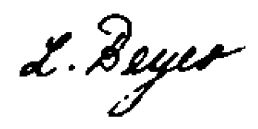

Lothar Beyer

\section{Korrespondenzadresse}

Prof. Dr. L. Beyer

Ärztehaus Mitte

Westbahnhofstraße 2, 07745 Jena, Deutschland lobeyer@t-online.de

Interessenkonflikt. L. Beyer gibt an, dass kein Interessenkonflikt besteht.
Auch online Zugang zu allen Beiträgen Ihres Zeitschriftenabonnements

Wussten Sie, dass Sie als Abonnent*in dieser Zeitschrift automatisch Online-Zugriffsrechte auf das gesamte Beitragsarchiv haben?

Der Zugang zu Ihrer Online-Bibliothek ist im Abonnement Ihrer Zeitschrift inbegriffen. Für den Zugang müssen Sie sich lediglich einmal über www.springermedizin.de/register registrieren.

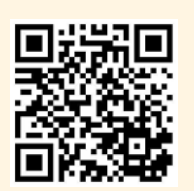

Über diesen QR-Code schnell und einfach registrieren

Bitte nutzen Sie für die Registrierung Vorund Nachname und Lieferadresse wie beim Abonnement der Zeitschrift (s. Adressaufkleber auf Ihrem Heft). So kann im System die Zugehörigkeit zu Ihrer Zeitschrift sichergestellt werden.

Aufgrund des Heilmittelwerbegesetzes dürfen die Inhalte der Website nur medizinischen Fachkreisen zur Verfügung gestellt werden. Bei der Anmeldung bitten wir Sie deshalb, einen Berufsnachweis vorzulegen. Bei einer Mitgliedschaft in der deutschen Ärztekammer reicht die Angabe der einheitliche Fortbildungsnummer (EFN). Alternativ schicken Sie eine Bestätigung des Arbeitgebers, Studiennachweis mit Angabe des Studiengangs ganz unkompliziert an kundenservice@springermedizin.de.

Mit Benutzername und Passwort haben Sie außerdem Zugang zu den freien Inhalten auf den Seiten von: www.springermedizin.de www.aerztezeitung.de

Sollten Fragen oder Probleme auftauchen, wenden Sie sich einfach an Ihren Kundenservice:

kundenservice@springermedizin.de 